\title{
Multi-pad Acupuncture Systems based on Internet Communication System
}

\author{
You-Sik Hong ${ }^{1}$, Sang-Suk Lee ${ }^{2}$ and Kyu-Tae Lee ${ }^{3}$ \\ ${ }^{1}$ Dept. of Computer Science, Sangji University, Wonju, Korea \\ ${ }^{2}$ Department of Oriental Biomedical Engineering, Sangji University, Wonju, Korea \\ ${ }^{3}$ DIvision of Information and communication Eng, Kongju National Univ., Korea
}

\begin{abstract}
In oriental medicine, patient's pulse wave signal has been used to estimate patients' health condition with it's speed, strength, and depth etc. However, even though the same doctor diagnose the patient, result could be different according to one's age, sex and body condition. In this paper, we developed a portable intelligent punctuate device for market release, which has a function of calculating punctuate time by setting proper information on the data. We expect this device could be used to remote location in everytime and everywhere.
\end{abstract}

Keywords: Inference system, fuzzy theory, acupuncture, pulse wave detection system, expert system

\section{Introduction}

This is a paper about studying of an intelligent punctuate system for using realtime body signal. The system's structure was figured out with following procedures. First, sensing block accept body signal as realtime to detect patient's condition. And then estimation block analyze the body signal, punctuate tip block shot a electric signal on the multi spot which has known as acupuncture point. On this research by using fuzzy estimation system, we could commit an error judging someone has heart disease just because of heart pulse rate is above 130. To resolve this kind of problem we made a constraint on the sensing system in case of drinking alchole, exercise, bath, anger and uneaseness. After simulation we found that the patient disease detecting system is well working even above 100 heart pulse per minute by fuzzy logic and fuzzy estimation rules [7-11].

In oriental medicine, the pulse beats are important signals that may let us know the conditions of one's health and disease [1-3]. In other words, doctors of oriental medicine can simply analyze pulse waves anywhere and anytime to treat patients without using high-priced medical appliances. However, they are largely subjective in interpreting the pulse rates and hence their reliability is far from being perfect. The current paper aims to solve this problem by using fuzzy inference rules in judging patients' health status and to develop a software kit of intelligent electronic needles. The physical characteristics including the thickness of skins and blood vessels should be also considered in order to analyze delicate pulse waves. We designed the system to respond to the various patterns and to sense the situation which potential difference is changed according to the patient's painful part simultaneously [4-7]. It contains the function that a patient can search the exact point of electronic acupuncture and check on optimal strength and time of electronic acupuncture considering the patient's body conditions. The patients can be treated with optimally, and acupuncture time can be shortened and strength of acupuncture can be moderated considering their condition. This system is useful for remote medical examination and treatment. It is genuinely difficult to produce an accurate analysis using the conventional pulse wave analyzing system. Recently, through the observation of the 
pulse, doctors have been able to distinguish the condition of blood vessels in seven types and can judge if a patient is ill or healthy. The present paper takes an individual patient's physical conditions into consideration in providing web-based medical treatment of electronic needles with an appeal to fuzzy inference [7-12]. In oriental medicine, the term 'pulse' has several meanings. The pulse is considered an important factor in herbal remedies, since observation of a person's pulse rate may reflect their health and illness. The observation of heart palpitations is critical, with the cessation of a person's heart palpitations classifying them as dead. Therefore, the condition of the instant heart change is observed in western medication and in eastern medication we can judge this by feeling the pulse. Since ancient times, oriental doctors have considered pulse rates as important data in diagnosis. Accordingly, a doctor who was competent at checking the pulse of their patient was considered a creditable doctor. However, the current blood pressure pulse analyzer may be considered flawed, since it is uncertain whether the blood pressure pulse analyzing sensor is located precisely on the radial artery. Furthermore, the analogue type blood pressure pulse analyzer has the issue of objectifying the blood pressure pulse. Although some people may have the same forearm length but the thickness of their blood vessel may differ and therefore there is no set of data that is considered reliable enough to judge the accuracy of blood pressure pulse rates. Therefore, in order to solve these kinds of problems, oriental doctors should not only judge the basic biological signals such as checking the pulse's size, strength, and speed, but should also consider the basic and quantitative analysis of the pulse in order to gain an accurate diagnosis. Also, the doctor should consider physical characteristics, such as the thickness of the skin and blood vessels, in order to reach an accurate conclusion. Therefore, measurement of the blood flow rate is a vital indicator in understanding the blood pressure rate and how the substances in the blood are transported. The composition of this paper is as follows: section 2 discusses the western and oriental medicine techniques; section 3 discusses the fuzzy rules based on pulse wave analysis. Section 4 reports the results of electronic needle simulation and section 5 concludes this paper.

\section{Pulse Wave Analyzing System}

Pulse wave is a record of pulse in chest wall and reat arteries which happens by heart palpitation and its main purpose is to observe the palpitation of heart and circulation of blood. Recently, the doctors are analyzing the patient's health condition through studying the blood circulation and changes in pulse wave. In other words, differentiating the pulse wave of the patient which is not stabilized nor has a distinct inflection point twice APG, the doctors are judging the patient's health condition in three states, positive which means safe, negative which means dangerous, and zero. During the first stage of studying the pulse, the pulse pressure was mainly used but recently due to simplicity of detection, volume pulse is being preferred. Second derivative of APG is a pulse wave that is derived from differentiating the volume pulse twice and was first recorded in 1972 by Ozawa of Japan. In 1978, Ozawa was observing the constant changes in accelerated APG as the patient's age increased. In this thesis, by analyzing the blood pressure pulse, we wish to accurately diagnose and verify the internal organs. When a patient is diagnosed with weak liver through checking the pulse, we should keep in mind that every person has a different physiological signal. In this case, we should verify this by electronically stimulating the hepatic pulse located on the patient's hand. If there is pain in liver when it is stimulated, then it can then be assured that the patient is suffering liver problem because when an area is wounded, the electric wave meets high resistance from the body. Therefore electric wave is weak in the wounded area and results in decrease of absolute current in tissue. 


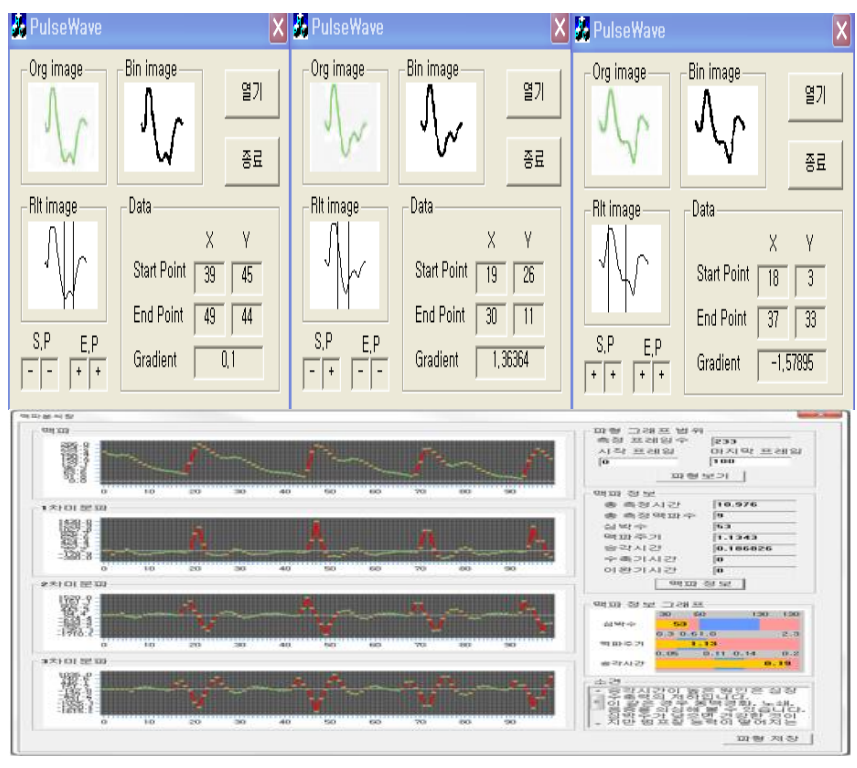

Figure 1. Blood Pressure Pulse Analyzing Test

In other words, when infection and various diseases such as cancer are found, then the person suffers pain, high fever, edema, and epilepsy. Therefore if a person is wounded or infected then the muscles naturally contract in order to protect the body. This is why the wounded area or the muscles contract, it results in blood flow reducing. Also, the bodily waste isn't excreted and piled which works as the main cause of muscle pain.

\section{Patient's Health Judgment using Fuzzy Rules}

This thesis has suggested the algorithm where the belief value regarding the disease affirmation is calculated, since we should take the patient's physical condition, age and gender into consideration. First, we should know the prior probability, $\mathrm{P}(\mathrm{Hj})$ and the conditional probability, $\mathrm{P}(\mathrm{E} \mid \mathrm{Hj})$ in order to get the conditional probability, $\mathrm{P}(\mathrm{Hi} \mid \mathrm{E})$. For example, $\mathrm{P}(\mathrm{E} \mid \mathrm{Hj})$, the probability that the symptom of each disease may upspring, should be given when it is inferred that $\mathrm{E}$ is the symptom with the patient's body and $\mathrm{Hi}$ is the disease. However, it is the reality that data for such things are insufficient quite often. Therefore, the belief value regarding disease affirmation is calculated, using fuzzy rule, as seen below.

Preconditioning RULE: (True Pulse)

IF S amp (amplitude) is maximum

OR S time of patient's pulse is maximum

AND N time of patient's pulse is maximum

AND R time of patient's pulse is maximum

OR R amp of patient's pulse is maximum

OR $\mathrm{N}$ amp of patient's pulse is maximum

Not patient's pulse is "Hongmaeck- a type of pulse"

Not patient's pulse is "Ginmaeck- a type of pulse" Not patient's pulse is "Noemaeck- a type of pulse" "

THEN it is true pulse. 


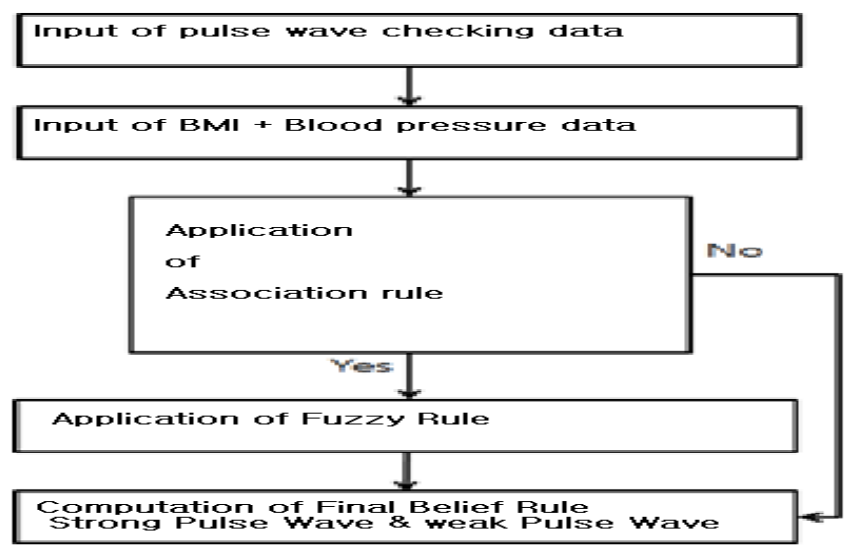

Figure 3. Flowchart of Intelligence Pulse wave System Using Fuzzy Rules

Figure 3 explains how the fuzzy rule and the belief value obtained through statisticbased probabilistic data are used in the final process for inference of disease if it is difficult to make a correct inference due to some physical conditions when more than 2 data of pulse wave checking and tongue diagnosis need to be inferred to measure the patient's disease more correctly.

\section{Rule: IF A is $\mathrm{t} 1$ THEN C is B2}

$(\mathrm{Fu})$

fact: A is t1' (Fr)

conclusion: $\mathrm{C}$ is $\mathrm{t} 2$ ' (FC)

A: Condition of clinical patient

C: Result of inference

Fu: fuzzy number showing the uncertainty of rule

Fr: fuzzy number showing the uncertainty of fact

FC: fuzzy number showing the uncertainty of conclusion

V1, V2, V1', V2': values

$\mathrm{Fu}$ shown above is represented by Confidence factor(CF) and $\mathrm{Fr}$ is represented by probability scale.

Post processing RULE (strong wave)

IF Samp $=$ Med And

$\mathrm{Bmi}=$ High And

H_Blood= High And

$\mathrm{N}$ time $=$ Med And

Then

Strong wave $=$ CNF 70

CNF mentioned herewith signifies that the certainty of strong (excess syndrome) wave rule is $70 \%$. The probability that a patient is diagnosed to be of strong wave is always $100 \%$, if we display the certainty factor, following the traditional method with no fuzzy rule included. The reliability of strong wave is counted to be $70 \%$ in this thesis, considering the H_Blood(maximal blood pressure), Samp(rise waveform) N time (notch 
point time) and $\mathrm{BMI}\left(\right.$ Body mass index; weight $\left.(\mathrm{kg}) / \mathrm{height} \exists \boldsymbol{} \exists\left(\mathrm{m}^{2}\right)\right)$. If a user gives the certainty of $80 \%$ to the fact that $\mathrm{S}$ amp = Low, the degree of certainty as regards the conclusion becomes $0.56(0.8 \times 0.7=0.56)$.

It is very difficult to extrapolate a disease from checking of pulse waveform because the velocity and intensity of pulse wave may differ depending on the gender, age and physical condition of patient. Therefore, this researcher used the belief value matching function which is to be used in recalculating belief value when there is more than 2 estimating values concerning same disease.

$\beta c=\beta \operatorname{comb}(\beta c, \beta$ strong $)(2)$

$=\max (\beta \mathrm{c}, \beta$ strong $)(3)$

' $\beta$ strong' shown herewith is the belief value of a conclusion that was already obtained through an inferring channel and ' $\beta \mathrm{c}$ 'is other belief value that was obtained through a different inferring channel. The data inputted from sensor regarding the strength of blood pressure is to be transformed into a suitable form for easy judgement through First Order Derivative and Second Order Derivative and the algorithm for data indexing is used in this process. Thus, the input data for fuzzy logic comes to be drawn up. The data drawn up in this way can be expressed in the form of IF-THEN'generally. The fuzzy inference is a succession of processes where a new relation or fact is inferred from some rule already given. And the max-min method of reasoning was used.

Input : $\mathrm{x}$ is $\mathrm{A} 1$ then $\mathrm{y}$ is $\mathrm{B}$

AND R1 : IF $\mathrm{x}$ is $\mathrm{A} 1 \mathrm{AND} \mathrm{y}$ is $\mathrm{B} 1$, THEN $\mathrm{z}$ is $\mathrm{C} 1$

OR R2 : IF $x$ is $A 2$ OR $y$ is $B 2$, THEN $z$ is $\mathrm{C} 2$

NOT $\mathrm{Rn}:$ IF $\mathrm{x}$ is An AND y is NOT Bn, THEN $\mathrm{z}$ is $\mathrm{Cn}$

Conclusion : $\mathrm{z}$ is $\mathrm{C}$ matching function

The belief value matching function is to be used in recalculating the belief value of a conclusion when more than 2 estimating values come into existence by fuzzy rule. For example, the probability that a patient does not have a substantial symptom(has an frail symptom) becomes 0.7 if the probability that a patient have a substantial symptom were 0.3. But, under some indefinite surroundings, the fuzzy scale does not allow us to affirm that the probability of frail symptom must be 0.7 if the probability of substantial symptom is 0.3 , It is because the probability of frail symptom might be $0.5,0.6$ or 0.7 since there are many variables such as physical condition, health situation, illness of patient and so on.

As a matter of fact, the oriental medicine has a so-called HEOSILBOSA(虛實補瀉) principle that the unenergetic should be complemented and the substantial should be scrubbed out. So, the word of exuberant pulse does not mean that one is full of vigor but that the wicked strength is vigorous in a patient who is resistible against a disease, as a disease broke into his body. On the other hand, the frail symptom means that a patient is lacking in vital force, has little resistance to a disease and become lacking in curative powers. In other words, the frail symptom is a nature of illness that was caused by insufficient vital force and the weakened health condition. And the substantial symptom can be interpreted to be a phenomenon where the function of some physical part is under the influence of abnormal exacerbation because the disease factor became very vigorous. Therefore, there are two types of prescription in Oriental Medicine; one is to strengthen the weakened part of human body in order to prevent and cure the disease of a patient and the other is to scrub out and expel the disease factor that is growing worse abnormally. 


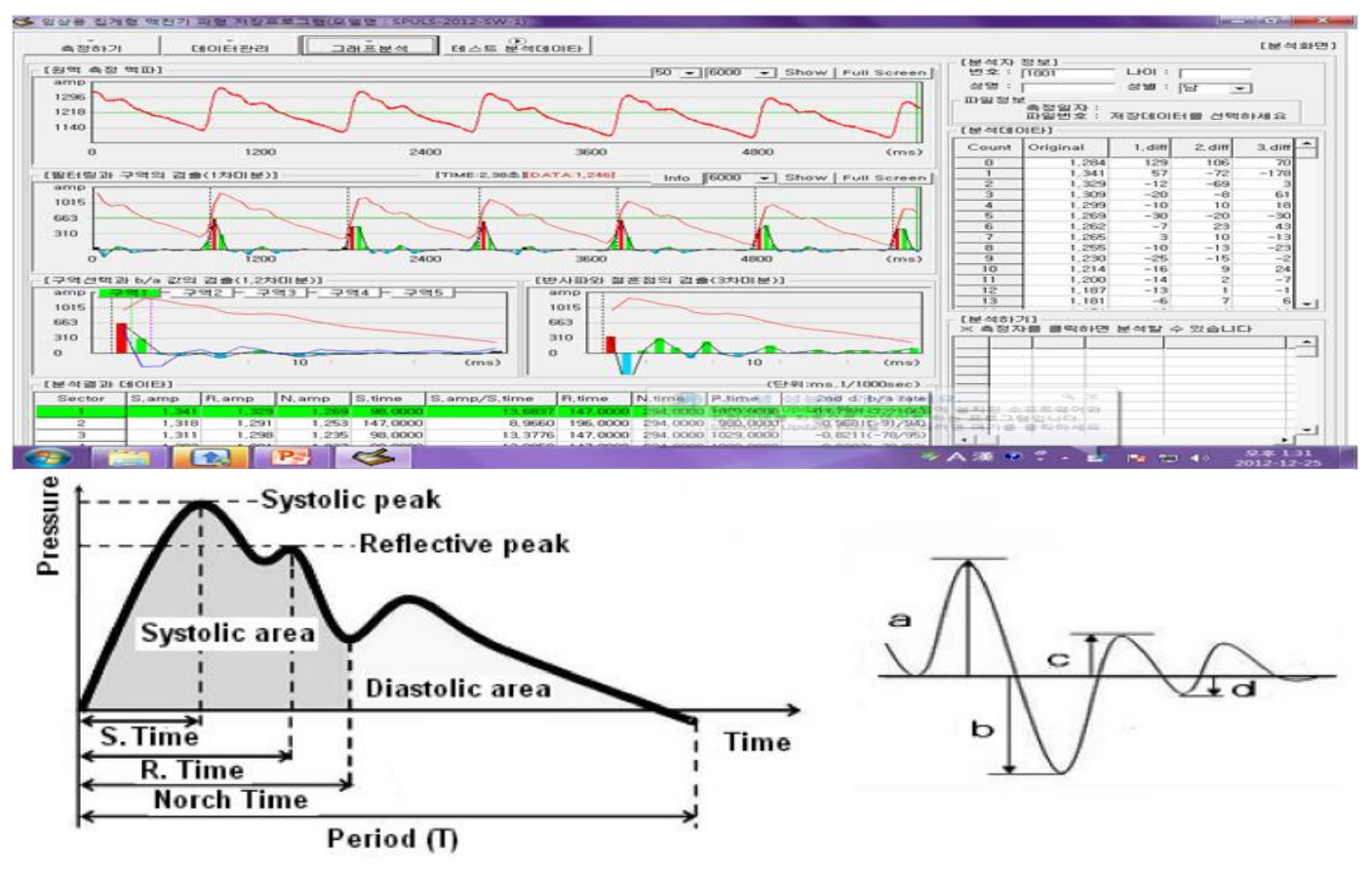

Figure 4. Explain for Arterial Pulse Wave of Pattient's Health Judgment

The following terms for the use of portable pulsimeter were shown in Figure 4.

(1) the amplitude value and time of pulse wave

(1) Systolic peak amplitude

(2) Reflective peak amplitude

(3) Notch amplitude

(4) Up-stroke time

(5) Slope of up-stroke

(6) Reflective wave time

(7) Notch time

(8) Total time

The researcher asked patients to respond to the questionnaire of self-reporting type that was translated from the "strong and weak pulse questionnaire", introduced in a journal of Japanese society of oriental medicine. At the same time, the researcher asked 5 oriental doctors to check this patients' pulse to certify whether the self-reported symptom coincides with the doctors' diagnosis regarding their pulse wave. Thus only those whose self-reporting has coincided with the doctors' pulse check were selected as the clinical test subjects. The clinical test started from the first subject chosen on 24 Sep. 2012 and ended with the last subject chosen on 14 Dec. 2012.

This research aimed at making an analysis of the exuberant pulse and the feeble pulse. So, 187 clinical patients were selected as the subjects of clinical test and it was judged whether they have the substantial symptom or frail symptom, which is regarded as an important index to check the patient's vitality in oriental medicine. The clinical patients were composed of 94 males and 93 females. 60 patients were turned out to belong to the frail symptom group, 64 were turned out to belong to the medium group and 63 were turned out to belong to the substantial symptom group. The procedures for clinical test are 
introduced hereunder.

The general algorithm comes to a conclusion of $\mathrm{B}$ on the assumption of 'A $\rightarrow$ B and equals A'. In this case the latter A should be completely consistent with the former A (of conditional clause). This kind of inference is called Modus Ponens and the Fuzzy algorithm is trying to expand as follows which will be called GMP (Generalized Modus Ponens);

Assumption 1: If $\mathrm{x}$ equals $\mathrm{A}, \mathrm{y}$ will be $\mathrm{B}$.

Assumption 2: $x$ equals A'.

Conclusion : y equals B'.

When you use the Fuzzy Rules, the knowledge to judge a patient's health by using the pulse wave will be expressed in Fuzzy R and you will be able to carry out modeling like the following to observe Output (or Symptom) B to clarify Input (or Cause) A.

If you are not careful for actual pulse feeling, often you may be confused that floating pulse, sliding pulse, and big pulse are powerful while submerging pulse and small pulse aren't. However, some floating pulse, big pulse, and sliding pulse are weak and some submerging pulse and small pulse are strong. The basic principles of the electronic acupuncture can be as follows. The human body displays pain, fever, edema and stroke if a certain spot suffers from infection or disease. Thus, the body or muscle tries to contract in order to protect itself from infection or injury on the skin or tissues of the body. As a consequence, the contracted muscle results in the decreased amount of blood current, oxygen and nutrients. In this paper, it developed a system of electronic acupuncture which automatically locates optimal spots and applies electronic needles.

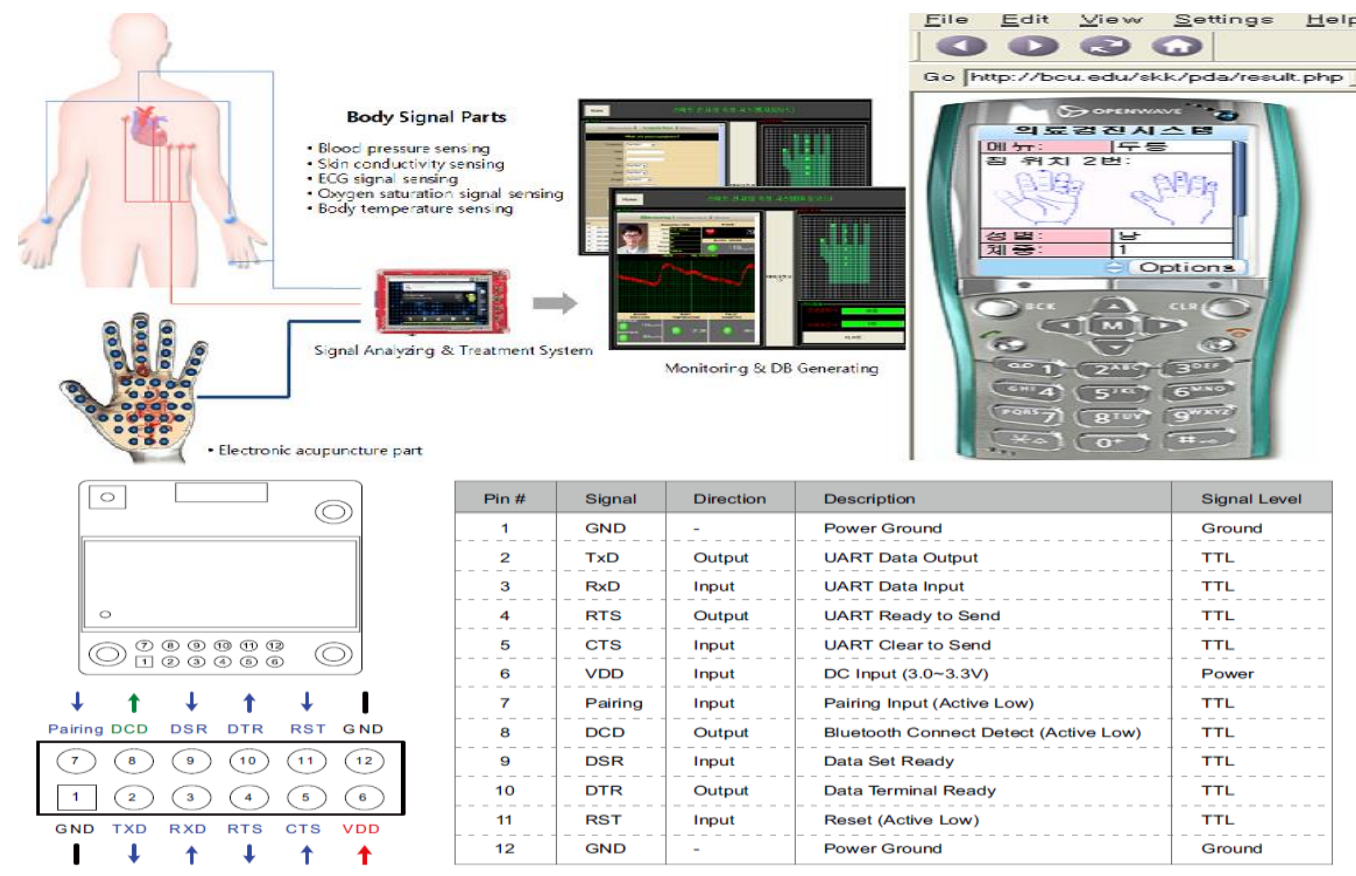

Figure 5. Electronic Acupuncture Simulation

Figure 5 shows the flow of the experiment. Multi electronic needles give stimuli to the reflex zones of a hand. The system senses where pain is felt and identifies the status of a patient's disease. Moreover, it shows the result of the web-based remote medical treatment of acupuncture. The conventional electronic needles can be categorized into two types: about $60 \%$ of them appeal to low frequency and the rest $40 \%$ utilize momentary electric stimuli plus needles. Low-frequency therapeutic apparatuses were introduced to the market, but they might be simply generators of low frequency $(16-32 \mathrm{~Hz})$. 


\section{Experimental Results}

What is a multi-pad with a built-active electronic-acupuncture depending on a patient's current body status? Based on this information, the patient meets the voltage and current self-oscillation. The frequency with the ability to automatically advanced procedure is called electronic-acupuncture. In order to perform these functions simultaneously with the sensing of electronic-acupuncture, one is required to possess the ability to perform surgery, derive accurate analysis from fuzzy logic and process statistical data. Electrical resistance of the body including long-term resistance, internal resistance and the surface can be divided into exposed skin. Resistance of the body when the DC voltage is based on the pure resistive component can be considered only based on the basis, when the impedance of the $\mathrm{AC}$ voltage should be considered. That body electrical conductors if you think skin, blood, muscles and other body each part of the voltage and current for the resistive component and capacity components are separated by impedance and its size, the electrical conduction path, the contact voltage, the contact area, and energizing time, is applied differently depending on the frequency may occur.
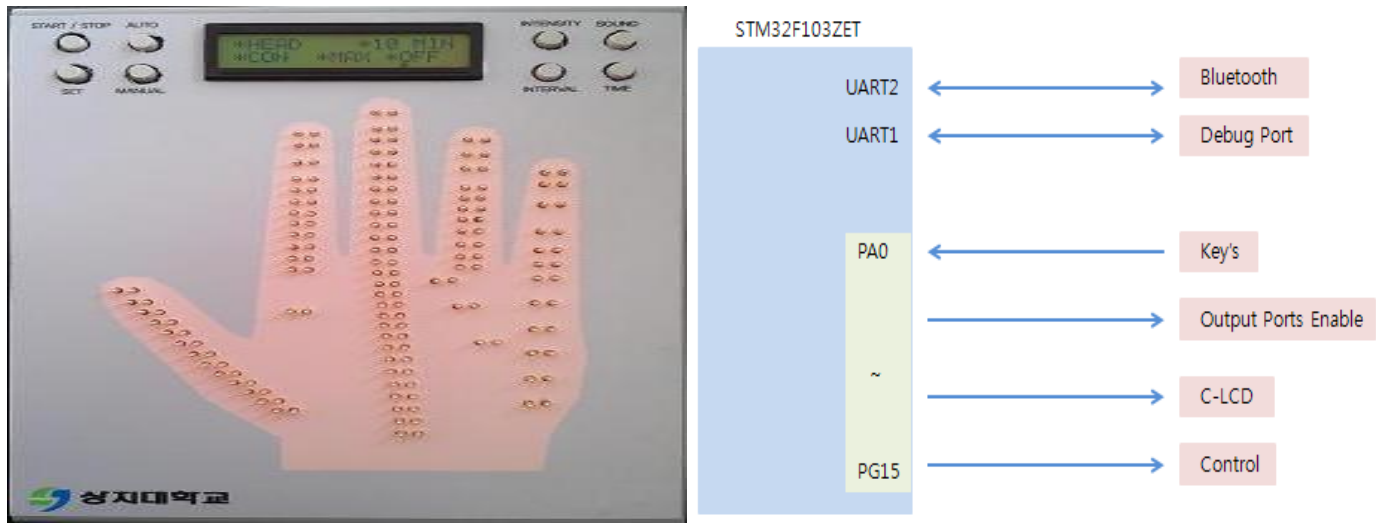

Figure 6. Multipad with a Built in Electronic Acupuncture

Figure 6 illustrates the basic theory of electronic-acupuncture. In addition, these changes in a person's age, gender, humidity, temperature, weight and fat accumulation is based on the changes. The requirements when considering the electrical resistance of human skin in general is based on the amount of approximately $2500 \Omega$. However, the same voltage and current is applied even if the amount of contact area and pain change in resistance over time are different. In the electrical resistance of human body tissues, regardless of the DC and AC power is almost constantly appear if time longer JUAL heat due to temperature rise of tissue resistance is slightly reduced. When the electricity in the human body typically conduct a minimum of power to feel the flow of the AC voltage is $1 \mathrm{~mA} \sim 2 \mathrm{~mA}$ for men. In contrast, direct the flow of power is smaller than the stimulus at least five double-road sensing current flow caused by the voltage applied, even though I do not feel the flow of electricity. Thus, in the treatment of electronic-acupuncture, electricity is AC rather than DC voltage with the voltage of the aneurysm and the frequency and voltage, over current change as a real hand acupuncture procedures, a small battery that has the same effect as a treatment is likely to be seen. In the experiment, according to AC current that can safely come off as self a man $16 \mathrm{~mA}(60 \mathrm{~Hz})$ women $10.5 \mathrm{~mA}(60 \mathrm{~Hz})$ is about the human body can withstand DC current is approximately $74 \mathrm{~mA}$ men for women is approximately $50 \mathrm{~mA}$. But it also including a person's body size and weight may appear slightly different depending on the requirements. In this paper, the voltage between $15 \mathrm{~V} \sim 50 \mathrm{~V}$ AC voltage to the change of $5 \mathrm{~Hz} \sim 1.2 \mathrm{Khz}$ and current $500 \mathrm{uA} \sim 1500 \mathrm{uA}$ given in the current experiments were carried out. If the fuzzy rules are not applied, the conventional method fixes the time of acupuncture at 15 minutes. 
However, this study classified the disease conditions into three stages: early, middle and terminal. For example, a patient in the early stage of disease is given 18 minutes according to the fuzzy rule if his or her height is High, age is Middle and weight is High. In this paper, it summarizes the pilot experiment of the real-time intelligent acupuncture system of controlling appropriate time and strength of acupuncture by using real-time information on the physical conditions of patients.

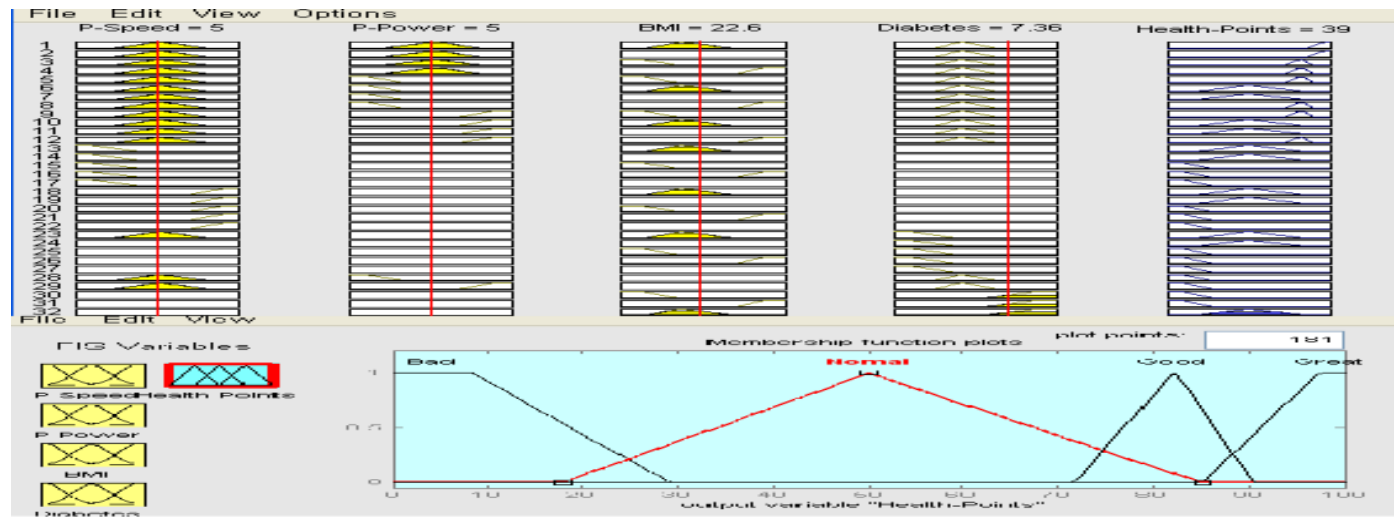

\section{Figure 7. Electronic Acupuncture Membership Functions and Rules using Fuzzy Rules}

Figure 7 explains the 32 fuzzy rules and fuzzy output membership. To take an example, if a patient input conditions $\mathrm{BMI}$ index $=22.6$, pulse speed $=5$, pulse power $=5$ and diabetes level=7 are health condition will be 39 .

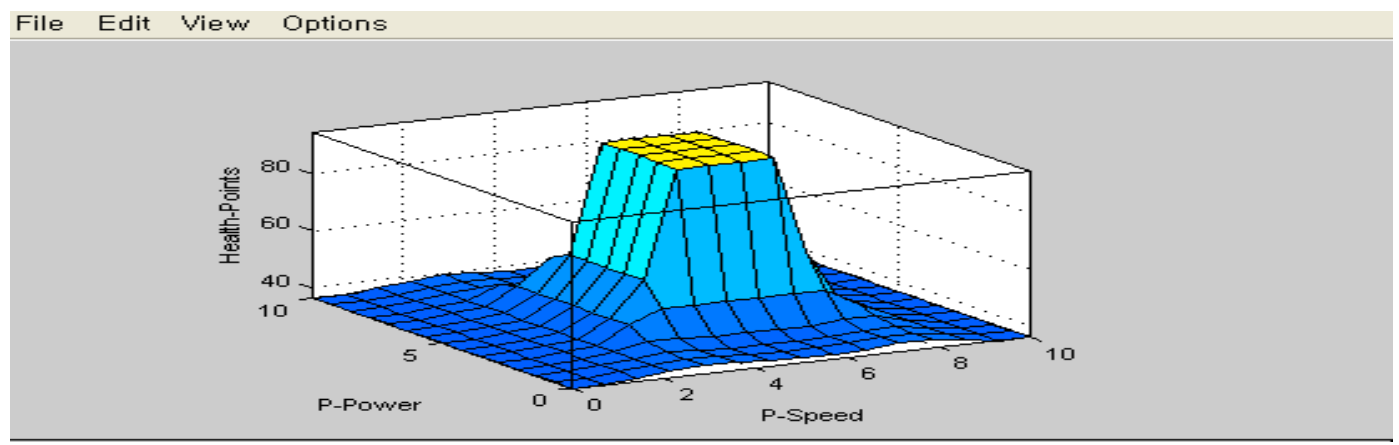

Figure 8. Electronic Acupuncture Output Simulation Using FIS MATLAB

Figure 5 displays the result of Matab Fuzzy inference system. It illustrates how the system calculates the output condition of time for acupuncture from the input data of the 4 conditions of a patient: physical conditions, skin impedance, age and status of disease. In this paper, in order to solve this kind of problem, it uses compositional inference while using the fuzzy rule. Fuzzy compositional rule of inference is applied to come up with a calibrating constant in order to derive an accurate result (considering the patient's physical condition) in analyzing the blood pressure pulse. In existing method, an oriental doctor infers one pulse wave out of 28 pulse wave and diagnoses the patient. Fuzzy compositional rule of inference is a rule made in order to come up with an inference by using fuzzy production rule which includes fuzzy variables. The form of fuzzy compositional rule of inference is as in the following.

Belief of fuzzy composite function: If fuzzy compositional rule of inference is applied in fuzzy production rule then belief of fuzzy evaluation function can't be used. So in order to calculate the belief of fuzzy function, the belief of fuzzy composite function is used. 


$$
\begin{aligned}
\beta c & =\beta \operatorname{comp}(\beta(\beta \mathrm{f}, \beta r)) \\
= & \min (\max (\beta(\beta \mathrm{fp}, \beta \mathrm{rpq})))
\end{aligned}
$$

In here, $\mathrm{p}=1,2, \cdots, \mathrm{m}, \mathrm{q}=1,2, \cdots, \mathrm{n} . \mathrm{m}, \mathrm{n}$ are the number of each fuzzy thesis in premise and conclusion. Belief of fuzzy union function in type 1 and 2 of fuzzy production rule which are the minimized version of type 5 and 6 can come up with the same node or conclusion using different inference. In this kind of node, same conclusion has two or more different belief of function. In this type of situation, in order to recalculate the belief of fuzzy function, fuzzy union function is used.

\section{Conclusion}

The DSP board, intelligent pulse decision system, inferred on this paper was designed to react small body signal of patient with different kinds of pattern and detect all the state of alternation. When inflammation or disease like cancer has detected, human body go through pain, spot heat or paroxysm. This leads to shrink of muscle, and shrink muscle block to flow of blood and oxygen including nutrition. And body waste stacked on the body without evacuation. As a result body muscle go through pain.

This theory reveal the fact that dark spot, acne, and atopy where has high electronic resistance that block the electronic current, cause skin lack of oxygen and finally leads to skin disorder. In other words, inflammation and death skin cause skin trouble. Therefore, this paper suggest an intelligent algorithm to cure the disease after detecting the patient pain region by themselves with portable WEB based multipad electric shot that could be used anywhere, anytime and for anyone.

In this paper, we proposed the system that diagnoses a patient optimally considering the patient's condition using intelligent fuzzy technique. We analyzed the fine distinction considering thickness of skin and blood vessels and pulse, weather big or small, strong or weak and fast or slow. We classified the patients by their body, illness and age, and calculated the exact time of electronic acupuncture suitable for the patient's physical condition using fuzzy logic and inference. Furthermore, by referring to interrelationship and index of similarity, oriental doctors are able to diagnose a patient with high/low blood pressure, diabetes, and cardiovascular disease. But in order to accurately analyze the pulse wave of a person, factors including height, weight, age, skin type, skin impedance, thickness of skin and blood vessels should be considered, since relying solely on the physical appearance of a human body may result in an incorrect interpretation of the disease. In this paper, it applied the fuzzy algorithm to analyze such a problem and draw an inference from objective data in view of pretreatment and applied the electrical characteristics of pain parts which respond to acupuncture device which considering intelligence pulse wave system using fuzzy rules.

\section{Acknowledgements}

This research was supported by the MSIP (Ministry of Science, ICT and Future Planning), Korea, under the IT/SW Creative research program supervised by the NIPA (National IT Industry Promotion Agency)" (NIPA-2013-H0502-13-1112).

\section{References}

[1] Y. J. Kim, "A meridian system study on Mawangtew medical books and Whang Di Nei Qing," The Journal of Oriental Medical Classics, vol. 9, (1995), pp. 51-86.

[2] H. G. Lee, "A study on meridian system using electrical characteristics," Korea Institute of Oriental Medicine, (1996).

[3] K. S. Soh, "Meridian point and meridian system in the Bong-Han Theory", (2003).

[4] Health Insurance plain seam, Statistical Yearbook of the Health Insurance Review and Assessment, Article 23, (2001). 
[5] K. S. Soh, "Meridian point and meridian system in the Bong-Han Theory", (2003).

[6] S. Finando and D. Finando, "Fascia and the mechanism of acupuncture", (2010).

[7] D. M. Jeong, "Hydrodynamic Analysis for Objectification of Meridian Substance by Vibration Stimulus of Body", Journal of the Korean Jungshin Science Society, vol. 3, no. 2, (1999), pp. 141-145.

[8] D. M. Jeong, "Implementation of the Visualizing System for Meridian by Overlapping with Bio-Signal and Image", Journal of the Korean Jungshin Science Society, vol. 3, no. 2, (1999), pp. 133-140.

[9] http://medi-core.co.kr/kor/apg.php.

[10] T. Whan Gwon, "The outlook of information and futere war," Korea National Defense University, (2004).

[11] Y. Sik Hong, "Medical Diagnosis System using Intelligence", The Journal of the Institute of Webcasting, Internet and Telecommunication, vol. 9, no. 1, (2009), pp. 25-30.

[12] H. You-Sik, "Smart Electro-acupuncture System", Proceedings of the Electronics Engineering, KAIST, (2011).

[13] National College of Oriental Medicine, Diagnostics, Saint functionality of Medicine, St Functional Medicine, Gunja publisher, (2007). 
International Journal of Bio-Science and Bio-Technology

Vol.6, No.4 (2014) 